\title{
Lower Bounds of Eigenvalues of the Stokes Operator by Nonconforming Finite Elements on Local Quasi-Uniform Grids
}

Youai $\mathrm{Li}^{*}$

School of Science, Beijing Technology and Business University, Beijing 100048, China

Received 27 February 2018; Accepted (in revised version) 18 October 2018

\begin{abstract}
This paper is a generalization of some recent results concerned with the lower bound property of eigenvalues produced by both the enriched rotated $Q_{1}$ and Crouzeix-Raviart elements of the Stokes eigenvalue problem. The main ingredient are a novel and sharp $L^{2}$ error estimate of discrete eigenfunctions, and a new error analysis of nonconforming finite element methods.
\end{abstract}

AMS subject classifications: 65N30, 65N15, 35J25

Key words: Lower bound, eigenvalue, nonconforming finite element method, Stokes operator.

\section{Introduction}

It was observed from numerical examples that some nonstandard finite element methods including nonconforming finite element methods and mass lumping finite element methods are able to yield lower bounds of eigenvalues of partial differential eigenvalue problems, see, Zienkiewicz et al. [32], for the Morley element, Rannacher [25], for the Morley and Adini elements, Liu and Yan [22], for the Wilson, enriched rotated $Q_{1}$, and rotated $Q_{1}$ elements. However, it was only very recent that these phenomena were rigorously analyzed. Indeed, Armentano and Duran [1] proposed an identity of errors of eigenvalues and proved that the Crouzeix-Raviart element produces lower bounds of eigenvalues for the Laplace operator provided that eigenfunctions $u \in H^{1+r}(\Omega) \cap H_{0}^{1}(\Omega)$ with $0<r<1$. The idea was generalized to the enriched rotated $Q_{1}$ element by the author of this paper in [14], and to the Wilson element in Zhang, Yang and Chen [31]. The extension to the Morley element was carried out in [29]. However, all of those papers are based on the saturation condition of finite element solutions by piecewise polynomials. The saturation condition can be from consistency errors and approximation errors as

*Corresponding author.

Email: lya@lsec.cc.ac.cn (Y. A. Li) 
well as regularity of exact solutions, and was proved based on a direct argument in $\mathrm{Hu}$, Huang and Lin [9]. If only the saturation condition of approximation errors is concerned, it was independently showed based on a contradiction argument in Lin, Xie and Xu [21]; see its application to nonconforming finite elements of the Laplace eigenvalue problem in [23]. In Hu, Huang and Lin [9], a more general result of the lower bound property of eigenvalues by nonconforming finite elements was established that if local approximation properties of nonconforming finite element spaces are better than total errors (sums of global approximation errors and consistency errors) of nonconforming finite element methods, corresponding methods will produce lower bounds for eigenvalues. For expansion methods based on superconvergence or extrapolation we refer interested readers to $[17,18,30,31]$, where the lower bound property of eigenvalues by nonconforming elements was analyzed on uniform rectangular meshes. We also refer interested readers to [10] for mass lumping finite element methods of eigenvalue problems.

The lower bound property of the eigenvalue by the nonconforming methods of the Stokes eigenvalue problem was first analyzed in [20], where a numerical result indicated that conforming finite elements of the Stokes eigenvalue problem are also possible to yield lower bounds of eigenvalues. In a recent paper by $\mathrm{Hu}$ and Huang [8], a more general framework is established for both conforming and nonconforming finite element methods for the Stokes operator. In particular, it was proved that the conforming $P_{2}-P_{0}$ element yields lower bounds of eigenvalues for the Stokes operator. However, all of these papers can only provide (asymptotic) lower bounds for eigenvalues on quasi-uniform grids.

In this paper we give a refined analysis for the lower bound property of eigenvalues by both the enriched rotated $Q_{1}$ [19] and Crouzeix-Raviart elements [9] of the Stokes eigenvalue problem. The main idea is to combine a series of new techniques: the elementwise Poincare-like inequality of the canonical interpolation operators of these two elements, a novel $L^{2}$ error estimate of discrete eigenfunctions from [8], a new error analysis of nonconforming finite element methods, plus the commuting property of the canonical interpolation operators.

In this paper, we use the standard gradient operator:

$$
\nabla r:=\left(\partial r / \partial x_{1}, \cdots, \partial r / \partial x_{n}\right)^{T} .
$$

Given any $n$ dimensional vector function $\psi=\left(\psi_{1}, \cdots, \psi_{n}\right)$, its divergence reads

$$
\operatorname{div} \psi:=\partial \psi_{1} / \partial x_{1}+\partial \psi_{2} / \partial x_{2}+\cdots+\partial \psi_{n} / \partial x_{n}
$$

The spaces $H_{0}^{1}(\Omega)$ and $L_{0}^{2}(\Omega)$ are defined as usual,

$$
\begin{aligned}
& H_{0}^{1}(\Omega):=\left\{v \in H^{1}(\Omega), v=0 \text { on } \partial \Omega\right\}, \\
& L_{0}^{2}(\Omega):=\left\{q \in L^{2}(\Omega), \int_{\Omega} q d x=0\right\} .
\end{aligned}
$$

This paper is organized as follows. In the next section, we present the Stokes eigenvalue problem. In Section 3, we get its nonconforming finite element methods. We present a 
new error analysis of nonconforming finite element methods in Section 4. In Section 5, we give a sharp $L^{2}$ error estimate of the discrete velocity and prove the final lower bound property of the discrete eigenvalues. We present the numerical experiments in Section 6.

\section{The Stokes eigenvalue problem}

The Stokes eigenvalue problem is defined as follows: Find $(\lambda, u, p) \in \mathbb{R} \times V \times Q:=\mathbb{R} \times$ $H_{0}^{1}(\Omega)^{n} \times L_{0}^{2}(\Omega)$ such that

$$
a(u, v)+b(v, p)+b(u, q)=\lambda(u, v) \quad \text { and }\|u\|=1 \text { for any }(v, q) \in V \times Q,
$$

where the bilinear forms $a(u, v)$ and $b(v, q)$ are defined as, respectively,

$$
a(u, v):=(\nabla u, \nabla v) \text { and } b(v, q):=-(\operatorname{div} v, q) .
$$

The kernel space of the divergence operator consists of all divergence free functions in $V$, which reads

$$
V_{0}:=\{v \in V, b(v, q)=0 \text { for any } q \in Q\} .
$$

Let $(\lambda, u, p)$ be the solution of the problem (2.1). It follows from (2.1) that $u \in V_{0}$ and that

$$
a(u, v)=\lambda(u, v) \quad \text { for any } v \in V_{0} .
$$

Then, we have that the eigenvalue problem (2.1) has a sequence of eigenvalues

$$
0<\lambda_{1} \leq \lambda_{2} \leq \lambda_{3} \leq \cdots \nearrow+\infty,
$$

and corresponding eigenfunctions

$$
\left(u_{1}, p_{1}\right),\left(u_{2}, p_{2}\right),\left(u_{3}, p_{3}\right), \cdots,
$$

which can be chosen to satisfy

$$
\left(u_{i}, u_{j}\right)=\delta_{i j}, \quad i, j=1,2, \cdots
$$

We define

$$
E_{\ell}:=\operatorname{span}\left\{u_{1}, u_{2}, \cdots, u_{\ell}\right\}
$$

Then, the eigenvalues and eigenfunctions satisfy the following well-known minimummaximum principle:

$$
\lambda_{k}=\min _{\operatorname{dim} V_{k}=k, V_{k} \subset V_{0}} \max _{v \in V_{k}} \frac{a(v, v)}{(v, v)}=\max _{u \in E_{k}} \frac{a(u, u)}{(u, u)} .
$$

\section{Two stable nonconforming finite element methods}

In this section we introduce two stable nonconforming finite elements from [19] and [9], respectively. 


\subsection{Enriched rotated $Q_{1}$ elements in any dimension}

Let $\mathcal{T}_{h}$ be regular $n$-rectangular triangulations of domains $\Omega \subset \mathbb{R}^{n}$ with $2 \leq n$ in the sense that $\bigcup_{K \in \mathcal{T}_{h}} K=\bar{\Omega}$, two distinct elements $K$ and $K^{\prime}$ in $\mathcal{T}_{h}$ are either disjoint, or share an $\ell$-dimensional hyper-plane, $\ell=0, \cdots, n-1$. Let $\mathcal{H}_{h}$ denote the set of all $n-1$ dimensional hyper-planes in $\mathcal{T}_{h}$ with the set of interior $n-1$ dimensional hyper-planes $\mathcal{H}_{h}(\Omega)$ and the set of boundary $n-1$ dimensional hyper-planes $\mathcal{H}_{h}(\partial \Omega) . \mathcal{N}_{h}$ is the set of nodes of $\mathcal{T}_{h}$ with the set of internal nodes $\mathcal{N}_{h}(\Omega)$ and the set of boundary nodes $\mathcal{N}_{h}(\partial \Omega)$.

For each $K \in \mathcal{T}_{h}$, introduce the following affine invertible transformation

$$
F_{K}: \hat{K} \rightarrow K, \quad x_{i}=h_{x_{i}, K} \xi_{i}+x_{i}^{0},
$$

with the center $\left(x_{1}^{0}, x_{2}^{0}, \cdots, x_{n}^{0}\right)$ and the lengths $2 h_{x_{i}, K}$ of $K$ in the directions of the $x_{i}$-axis, and the reference element $\hat{K}=[-1,1]^{n}$. In addition, set $h=\max _{1 \leq i \leq n} h_{x_{i}}$.

Denote by $\mathrm{EQ}(K)$ the enriched rotated $Q_{1}$ element space defined by [19]

$$
\mathrm{EQ}(K):=P_{1}(K)+\operatorname{span}\left\{x_{1}^{2}, x_{2}^{2}, \cdots, x_{n}^{2}\right\} .
$$

The enriched rotated $Q_{1}$ element space $V_{h}^{E Q}$ is then defined by

$$
\begin{aligned}
V_{h}^{E Q}:=\left\{v \in L^{2}(\Omega)^{n}:\left.v\right|_{K} \in \mathrm{EQ}(K)^{n} \text { for each } K \in \mathcal{T}_{h}, \int_{E}[v] d E=0,\right. \\
\\
\quad \text { for all internal } n-1 \text { dimensional hyper-planes } E, \\
\left.\quad \text { and } \int_{E} v d E=0 \text { for all } E \text { on } \partial \Omega\right\} .
\end{aligned}
$$

Here and throughout this paper, $[v]$ denotes the jump of $v$ across $E$.

\subsection{Enriched Crouzeix-Raviart elements in any dimension}

Suppose that $\bar{\Omega}$ is covered exactly by shape-regular partitions $\mathcal{T}_{h}$ consisting of $n$-simplices in $n$ dimensions. Given $E \in \mathcal{H}_{h}$, let $v$ be unit normal vector.

To obtain a nonconforming finite element method that is able to produce lower bounds of eigenvalues of second order elliptic operators, it was proposed in [9] to enrich the shape function space $P_{1}(K)$ by span $\left\{\sum_{i=1}^{n} x_{i}^{2}\right\}$ on each element. This leads to the following shape function space

$$
\operatorname{ECR}(K):=P_{1}(K)+\operatorname{span}\left\{\sum_{i=1}^{n} x_{i}^{2}\right\} \quad \text { for any } K \in \mathcal{T} .
$$

The enriched Crouzeix-Raviart element space $V_{h}^{E C R}$ is then defined by

$$
\begin{aligned}
V_{h}^{E C R}:=\left\{v \in L^{2}(\Omega)^{n}:\left.v\right|_{K} \in \operatorname{ECR}(K)^{n} \text { for each } K \in \mathcal{T}, \int_{E}[v] d E=0,\right. \\
\left.\quad \text { for all } E \in \mathcal{H}_{h}(\Omega), \text { and } \int_{E} v d E=0 \text { for all } E \in \mathcal{H}_{h}(\partial \Omega)\right\} .
\end{aligned}
$$


The discrete pressure space $Q_{h}$ is defined by

$$
Q_{h}:=\left\{q \in Q,\left.q\right|_{K} \in P_{0}(K) \text { for any } K \in \mathcal{T}_{h}\right\},
$$

where $P_{\ell}(K)$ denote the space of polynomials of degree $\leq \ell$.

In this paper, we take the nonconforming finite element spaces $V_{h}^{n c}$ as $V_{h}^{E Q}$ or $V_{h}^{E C R}$. Then, the discrete eigenvalue problem is to seek $\left(\lambda_{h}, u_{h}, p_{h}\right) \in \mathbb{R} \times V_{h}^{n c} \times Q_{h}$ such that $\left\|u_{h}\right\|=$ 1 and that

$$
a_{h}\left(u_{h}, v_{h}\right)+b_{h}\left(v_{h}, p_{h}\right)+b_{h}\left(u_{h}, q_{h}\right)=\lambda_{h}\left(u_{h}, v_{h}\right) \quad \text { for all } \quad\left(v_{h}, q_{h}\right) \in V_{h}^{n c} \times Q_{h} .
$$

We define the semi-norm over $V_{h}^{n c}+V$ by $\|\cdot\|_{h}:=a_{h}(\cdot, \cdot)^{1 / 2}$, it follows from the definition of $V_{h}^{n c}$ that $\|\cdot\|_{h}$ is a norm over the discrete velocity space $V_{h}^{n c}$ under consideration.

We define the kernel space of the discrete divergence operator by

$$
V_{0, h}:=\left\{v_{h} \in V_{h}^{n c}, b_{h}\left(v_{h}, q_{h}\right)=0 \text { for any } q_{h} \in Q_{h}\right\} \text {. }
$$

Let $\left(\lambda_{h}, u_{h}, p_{h}\right)$ be the solution of the problem (3.5), we have $u_{h} \in V_{0, h}$ and

$$
a_{h}\left(u_{h}, v_{h}\right)=\lambda_{h}\left(u_{h}, v_{h}\right) \text { for any } v_{h} \in V_{0, h} .
$$

Let $N:=\operatorname{dim} V_{0, h}$. The discrete problem (3.5) admits a sequence of discrete eigenvalues

$$
0<\lambda_{1, h} \leq \lambda_{2, h} \leq \cdots \leq \lambda_{N, h}
$$

and corresponding eigenfunctions

$$
\left(u_{1, h}, p_{1, h}\right),\left(u_{2, h}, u_{2, h}\right), \cdots,\left(u_{N, h}, p_{N, h}\right) .
$$

In the case where $\left(V_{h}, Q_{h}\right)$ is an conforming approximation in the sense $V_{0, h} \subset V_{0}$, it immediately follows from the minimum-maximum principle (2.3) that

$$
\lambda_{k} \leq \lambda_{k, h}, \quad k=1,2, \cdots, N
$$

which indicates that $\lambda_{k, h}$ is an approximation above to $\lambda_{k}$.

We define the discrete counterpart of $E_{\ell}$ by

$$
E_{\ell, h}:=\operatorname{span}\left\{u_{1, h}, u_{2, h}, \cdots, u_{\ell, h}\right\}
$$

Then, we have the following discrete minimum-maximum principle:

$$
\lambda_{k, h}=\min _{\operatorname{dim} V_{k, h}=k, V_{k, h} \subset V_{0, h}} \max _{v \in V_{k, h}} \frac{a_{h}(v, v)}{(v, v)}=\max _{u \in E_{k, h}} \frac{a_{h}(u, u)}{(u, u)} .
$$




\section{A new error analysis of nonconforming finite element methods}

For the analysis, we need a new error analysis of nonconforming finite element methods of the Stokes problem, which can be regarded as the generalization to the current case of those results from $[6,11,16,24]$.

Consider the Stokes problem: Given $f \in L^{2}(\Omega)$ find $(u, p) \in V \times Q$ such that

$$
a\left(u_{f}, v\right)+b\left(v, p_{f}\right)+b\left(u_{f}, q\right)=(f, v) \quad \text { for any }(v, q) \in V \times Q .
$$

The discrete problem of (4.1) is to seek $\left(u_{f, h}, p_{f, h}\right) \in V_{h} \times Q_{h}$ such that

$$
a_{h}\left(u_{f, h}, v_{h}\right)+b_{h}\left(v_{h}, p_{f, h}\right)+b_{h}\left(u_{f, h}, q_{h}\right)=\left(f, v_{h}\right) \quad \text { for all } \quad\left(v_{h}, q_{h}\right) \in V_{h} \times Q_{h} .
$$

We need some quasi-interpolation/approximation operator $\pi_{h}: V_{h}^{n c} \rightarrow V_{h}^{c}$, where $V_{h}^{c} \subset$ $H_{0}^{1}(\Omega)$ is some conforming finite element space over the triangulation $T_{h}$. We take $V_{h}^{c}$ as the conforming linear finite element space for the triangulation mesh and the conforming n-linear finite element space for the quadrilateral mesh. Given $v_{h} \in V_{h}^{n c}$, the interpolation function $\pi_{h} v_{h} \in V_{h}^{c}$ is defined as

$$
\begin{aligned}
& \left(\pi_{h} v_{h}\right)(\mathcal{P}):=\frac{1}{\left|\omega_{\mathcal{P}}\right|} \int_{\omega_{\mathcal{P}}} v_{h} d \sigma \text { for any interior vertex } \mathcal{P}, \\
& \left(\pi_{h} v_{h}\right)(\mathcal{P}):=0 \text { for any boundary vertex } \mathcal{P},
\end{aligned}
$$

where $\omega_{\mathcal{P}}$ is the node patch defined as $\omega_{\mathcal{P}}=\left\{K \in T_{h}, \mathcal{P}\right.$ is a vertex of $\left.K\right\}$. Such a kind of operators was proposed in [26]. For this quasi-interpolation operator, we have the following properties, whose proof can be found in, for instance, [2].

Lemma 4.1. There holds for any $v_{h} \in V_{h}^{n c}$ and $K \in T_{h}$ that

$$
\begin{aligned}
& \left\|v_{h}-\pi_{h} v_{h}\right\|_{0, K} \lesssim h_{K}\left\|\nabla_{h} v_{h}\right\|_{0, \omega_{K}} \\
& \left\|v_{h}-\pi_{h} v_{h}\right\|_{0, E} \lesssim h_{E}^{\frac{1}{2}}\left\|\nabla_{h} v_{h}\right\|_{0, \omega_{E}} \\
& \left\|\nabla_{h}\left(v_{h}-\pi_{h} v_{h}\right)\right\|_{0, \Omega} \lesssim\left\|\nabla_{h} v_{h}\right\|_{0, \Omega} .
\end{aligned}
$$

Here and throughout the paper, we shall use the notation

$$
A_{1} \lesssim B_{1} \quad \text { and } \quad A_{2} \approx B_{2}
$$

to denote that there exist constants $C_{1}, c_{2}$ and $C_{2}$ such that

$$
A_{1} \leq C_{1} B_{1} \quad \text { and } \quad c_{2} B_{2} \leq A_{2} \leq C_{2} B_{2}
$$

Before we present a new error analysis of the approximate solution $\left(u_{f, h}, p_{f, h}\right)$, we need the following result. 
Lemma 4.2. Let $\left(u_{f}, p_{f}\right)$ be the solution of Problem (4.1). Then, it holds, for any $s_{h} \in V_{h}^{n c}$ and $q_{h} \in Q_{h}, K \in T_{h}$, and $E \in \mathcal{E}$, that

$$
\begin{aligned}
& h_{K}\left\|f+\Delta s_{h}+\nabla q_{h}\right\|_{0, K} \lesssim\left\|\nabla\left(u_{f}-s_{h}\right)\right\|_{0, K}+\left\|p_{f}-q_{h}\right\|_{0, K}+h_{K}\|f-w\|_{0, K} \\
& h_{E}^{\frac{1}{2}}\left\|\left[\frac{\partial s_{h}}{\partial v}+q_{h} v\right]\right\|_{0, E} \lesssim\left\|\nabla_{h}\left(u_{f}-s_{h}\right)\right\|_{0, \omega_{E}}+\left\|p_{f}-q_{h}\right\|_{0, \omega_{E}}+\sum_{K \in \omega_{E}} h_{E}\|f-w\|_{0, K},
\end{aligned}
$$

for any $w \in P_{2}(K)^{2}$

Proof. We will use the bubble function technique from [28]. We first prove the efficiency of $\left\|f+\Delta s_{h}+\nabla q_{h}\right\|_{0, K}$.

$$
\begin{aligned}
\left\|f+\Delta s_{h}+\nabla q_{h}\right\|_{0, K}^{2} & =\left\|f-w+w+\Delta s_{h}+\nabla q_{h}\right\|_{0, K}^{2} \\
& \lesssim\|f-w\|_{0, K}^{2}+\left\|w+\Delta s_{h}+\nabla q_{h}\right\|_{0, K}^{2} .
\end{aligned}
$$

Let $\lambda_{i}, i=1,2,3$, be the barycenter coordinate of the triangle $K$. Let $v_{K}=b_{K}\left(w+\Delta_{h} s_{h}+\nabla q_{h}\right)$ with the element bubble function $b_{K}=27 \lambda_{1} \lambda_{2} \lambda_{3}$. Let $i d$ be an identity matrix. We consider the following term

$$
\begin{aligned}
& \left\|w+\Delta s_{h}+\nabla q_{h}\right\|_{0, K}^{2} \\
\approx & \left(b_{K}\left(w+\Delta s_{h}+\nabla q_{h}\right), w+\Delta s_{h}+\nabla q_{h}\right)_{0, K} \\
= & \left(v_{K}, w-f\right)_{0, K}+\left(v_{K}, f+\Delta s_{h}+\nabla q_{h}\right)_{0, K} \\
= & \left(v_{K}, w-f\right)_{0, K}+\left(\nabla u_{f}+p_{f} i d, \nabla v_{K}\right)_{0, K}+\left(v_{K}, \Delta s_{h}+\nabla q_{h}\right)_{0, K} \\
= & \left(v_{K}, w-f\right)_{0, K}+\left(\left(\nabla u_{f}-\nabla s_{h}\right)+\left(p_{f}-q_{h}\right) i d, \nabla v_{K}\right)_{0, K} \\
\leq & \left\|v_{K}\right\|_{0, K}\|w-f\|_{0, K}+\left(\left\|\nabla\left(u_{f}-s_{h}\right)\right\|_{0, K}+\left\|p_{f}-q_{h}\right\|_{0, K}\right)\left\|\nabla v_{K}\right\|_{0, K} .
\end{aligned}
$$

This inequality and the inverse estimate

$$
\left\|\nabla v_{K}\right\|_{0, K} \lesssim h_{K}^{-1}\left\|v_{K}\right\|_{0, K}
$$

lead to

$$
\left\|w+\Delta s_{h}+\nabla q_{h}\right\|_{0, K} \lesssim\|w-f\|_{0, K}+h_{K}^{-1}\left(\left\|\nabla\left(u_{f}-s_{h}\right)\right\|_{0, K}+\left\|p_{f}-q_{h}\right\|_{0, K}\right)
$$

which proves (4.4a).

We turn to $(4.4 b)$. Set

$$
v_{E}=\left[\frac{\partial s_{h}}{\partial v}+q_{h} v\right] b_{E}
$$

with the usual edge bubble function $b_{E}$ from [28]. Then, the Poincare inequality and the 
inverse estimate give

$$
\begin{aligned}
& \left\|\left[\frac{\partial s_{h}}{\partial v}+q_{h} v\right]\right\|_{0, E}^{2} \\
\approx & \left(b_{E}\left[\frac{\partial s_{h}}{\partial v}+q_{h} v\right],\left[\frac{\partial s_{h}}{\partial v}+q_{h} v\right]\right)_{0, E} \\
= & \left(v_{E},\left[\frac{\partial s_{h}}{\partial v}+q_{h} v\right]\right)_{0, E} \\
= & \left(\nabla_{h} s_{h}+q_{h} i d, \nabla v_{E}\right)_{0, \omega_{E}}+\left(v_{E}, \Delta_{h} s_{h}+\nabla_{h} q_{h}\right)_{0, \omega_{E}} \\
= & \left(\nabla_{h}\left(s_{h}-u_{f}\right)+\left(q_{h}-p_{f}\right) i d, \nabla v_{E}\right)_{0, \omega_{E}}+\left(\nabla u_{f}+p_{f} i d, \nabla v_{E}\right)_{0, \omega_{E}}+\left(\Delta_{h} s_{h}+\nabla_{h} q_{h}, v_{E}\right)_{0, \omega_{E}} \\
\leq & \left(\left\|\nabla_{h}\left(s_{h}-u_{f}\right)\right\|_{0, \omega_{E}}+\left\|q_{h}-p_{f}\right\|_{0, \omega_{E}}\right)\left\|\nabla_{h} v_{E}\right\|_{0, \omega_{E}}+\left\|f+\Delta_{h} s_{h}+\nabla_{h} q_{h}\right\|_{0, \omega_{E}}\left\|v_{E}\right\|_{0, \omega_{E}} \\
\lesssim & \left(\left\|\nabla_{h}\left(s_{h}-u_{f}\right)\right\|_{0, \omega_{E}}+\left\|q_{h}-p_{f}\right\|_{0, \omega_{E}}+h_{E}\left\|f+\Delta_{h} s_{h}+\nabla_{h} q_{h}\right\|_{0, \omega_{E}}\right) h_{E}^{-\frac{1}{2}}\left\|\left[\frac{\partial s_{h}}{\partial v}\right]\right\|_{0, E^{\prime}}
\end{aligned}
$$

which implies

$$
h_{E}^{\frac{1}{2}}\left\|\left[\frac{\partial s_{h}}{\partial v}+q_{h} v\right]\right\|_{0, E} \lesssim\left\|\nabla_{h}\left(s_{h}-u_{f}\right)\right\|_{0, \omega_{E}}+\left\|q_{h}-p_{f}\right\|_{0, \omega_{E}}+h_{E}\left\|f+\Delta_{h} s_{h}+\nabla_{h} q_{h}\right\|_{0, \omega_{E}} .
$$

This completes the proof.

Theorem 4.1. Let $\left(u_{f}, p_{f}\right)$ and $\left(u_{f, h}, p_{f, h}\right)$ be the solutions of Problems (4.1) and (4.2), respectively. Then, it holds that

$$
\begin{aligned}
& \left\|\nabla_{h}\left(u_{f}-u_{f, h}\right)\right\|_{0}+\left\|p_{f}-p_{f, h}\right\|_{0} \\
\lesssim & \inf _{v_{h} \in V_{h}^{n c}}\left\|\nabla_{h}\left(u_{f}-v_{h}\right)\right\|_{0}+\inf _{q_{h} \in Q_{h}}\left\|p_{f}-q_{h}\right\|_{0}+\left(\sum_{K \in \mathcal{T}_{h}} h_{K}^{2} \inf _{w \in P_{2}(K)^{2}}\|f-w\|_{0, K}^{2}\right)^{1 / 2} .
\end{aligned}
$$

Proof. By the theories of mixed finite elements and nonconforming finite elements, see for instance, $[3,4]$, it follows that

$$
\begin{aligned}
& \left\|\nabla_{h}\left(u_{f}-u_{f, h}\right)\right\|_{0}+\left\|p_{f}-p_{f, h}\right\|_{0} \\
\lesssim & \inf _{v_{h} \in V_{h}^{n c}}\left\|\nabla_{h}\left(u_{f}-v_{h}\right)\right\|_{0}+\inf _{q_{h} \in Q_{h}}\left\|p_{f}-q_{h}\right\|_{0}+\sup _{0 \neq v_{h} \in V_{h}^{n c}} \frac{a_{h}\left(u_{f}, v_{h}\right)+b_{h}\left(v, p_{f}\right)-\left(f, v_{h}\right)}{\left\|\nabla_{h} v_{h}\right\|_{0}} .
\end{aligned}
$$

Next we shall bound the consistent error term on the right-hand side of (4.8) by the approximation error term plus a oscillation term (up to some multiplicative constant). In fact, for any $v_{h}, s_{h} \in V_{h}^{n c}$, it follows from (4.1) that

$$
\begin{aligned}
& a_{h}\left(u_{f}, v_{h}\right)+b_{h}\left(v_{h}, p_{f}\right)-\left(f, v_{h}\right) \\
= & a_{h}\left(u_{f}, v_{h}-\pi_{h} v_{h}\right)+b_{h}\left(v_{h}-\pi_{h} v_{h}, p_{f}\right)-\left(f, v_{h}-\pi_{h} v_{h}\right) \\
= & a_{h}\left(u_{f}-s_{h}, v_{h}-\pi_{h} v_{h}\right)+b_{h}\left(v_{h}-\pi_{h} v_{h}, p_{f}-q_{h}\right) \\
& \quad-\left(f, v_{h}-\pi_{h} v_{h}\right)+a_{h}\left(s_{h}, v_{h}-\pi_{h} v_{h}\right)+b_{h}\left(v_{h}-\pi_{h} v_{h}, q_{h}\right) .
\end{aligned}
$$


By Lemma 4.1, the first and second term can be estimated as

$$
a_{h}\left(u_{f}-s_{h}, v_{h}-\pi_{h} v_{h}\right)+b_{h}\left(v_{h}-\pi_{h} v_{h}, p_{f}-q_{h}\right) \lesssim\left(\left\|\nabla_{h}\left(u_{f}-s_{h}\right)\right\|_{0}+\left\|p_{f}-q_{h}\right\|_{0}\right)\left\|\nabla_{h} v_{h}\right\|_{0} .
$$

After an integration by parts, the last three terms can be bounded by Lemma 4.1 and Lemma 4.2 as

$$
\begin{aligned}
& -\left(f, v_{h}-\pi_{h} v_{h}\right)+a_{h}\left(s_{h}, v_{h}-\pi_{h} v_{h}\right)+b_{h}\left(v_{h}-\pi_{h} v_{h}, q_{h}\right) \\
\lesssim & \left\|\nabla_{h}\left(u_{f}-s_{h}\right)\right\|_{0}+\left\|p_{f}-q_{h}\right\|_{0}+\left(\sum_{K \in \mathcal{T}_{h}} h_{K}^{2} \inf _{w \in P_{2}(K)^{2}}\|f-w\|_{0, K}^{2}\right)^{1 / 2},
\end{aligned}
$$

which completes the proof.

\section{Lower bounds of eigenvalues}

We need to bound the $L^{2}$ error of the discrete velocity. To this end, we follow [8] to introduce the quasi-Ritz-Galerkin projection $\left(\mathcal{P}_{h} u, \mathcal{P}_{h} p\right) \in V_{h}^{n c} \times Q_{h}$ as

$$
a_{h}\left(\mathcal{P}_{h} u, v_{h}\right)+b_{h}\left(v_{h}, \mathcal{P}_{h} p\right)+b_{h}\left(\mathcal{P}_{h} u, q_{h}\right)=\left(\lambda u, v_{h}\right) \quad \text { for any } \quad\left(v_{h}, q_{h}\right) \in V_{h}^{n c} \times Q_{h},
$$

where $(\lambda, u, p)$ is the eigenpair of Problem (2.1).

Lemma 5.1 (see [8]). Let $(\lambda, u, p)$ and $\left(\lambda_{h}, u_{h}, p_{h}\right)$ be the solutions of Problems (2.1) and (3.5), respectively. Then,

$$
\left\|u-u_{h}\right\|_{0} \leq 2(1+d)\left\|u-\mathcal{P}_{h} u\right\|_{0},
$$

where $d$ is the separation constant, see [8] for more details.

Let $\left(w_{d}, p_{d}\right)$ be the solution of the following dual problem: Find $\left(w_{d}, p_{d}\right) \in H_{0}^{1}(\Omega)^{n} \times$ $L_{0}^{2}(\Omega)$ such that

$$
a\left(w_{d}, v\right)+b\left(v, p_{d}\right)+b\left(w_{d}, q\right)=\left(u-\mathcal{P}_{h} u, v\right) \quad \text { for any }(v, q) \in H_{0}^{1}(\Omega)^{n} \times L_{0}^{2}(\Omega) .
$$

Assume the solution $\left(w_{d}, p_{d}\right)$ has the following regularity:

$$
\left\|w_{d}\right\|_{1+\sigma}+\left\|p_{d}\right\|_{\sigma} \lesssim\left\|u-\mathcal{P}_{h} u\right\|_{0},
$$

where $0<\sigma \leq 1$. Then a standard dual argument of nonconforming finite elements, see for instance, $[3,4]$, shows that

$$
\left\|u-\mathcal{P}_{h} u\right\|_{0} \lesssim h^{\sigma}\left\|\nabla_{h}\left(u-\mathcal{P}_{h} u\right)\right\|_{0} .
$$

For both the enriched rotated $Q_{1}$ and Crouzeix-Raviart elements, we can define the interpolation operator $\Pi_{h}: H_{0}^{1}(\Omega)^{n} \rightarrow V_{h}^{n c}$ by

$$
\begin{array}{ll}
\int_{E} \Pi_{h} v d E=\int_{E} v d E & \text { for any } v \in H_{0}^{1}(\Omega)^{n}, \quad E \in \mathcal{H}_{h}, \\
\int_{K} \Pi_{h} v d x=\int_{K} v d x & \text { for any } K \in \mathcal{T}_{h} .
\end{array}
$$


For this interpolation operator, since $u-\Pi_{h} u$ has vanishing mean on $K$, it follows from the Poincare inequality that

$$
\left\|u-\Pi_{h} u\right\|_{0, K} \leq C h_{K}\left\|\nabla\left(u-\Pi_{h} u\right)\right\|_{0, K} .
$$

Before state the second property of the interpolation operator, we introduce the following space, for any $K \in \mathcal{T}_{h}$,

$$
C_{K}^{E Q}=\left(\begin{array}{c}
a_{11}+a_{12} x_{1} \\
a_{21}+a_{22} x_{2} \\
\cdots \\
a_{n 1}+a_{n 2} x_{n}
\end{array}\right),
$$

for $a_{11}, a_{12}, \cdots, a_{n 1}, a_{n 2} \in \mathbb{R}$. Let $P_{K}^{E Q}$ be the $L^{2}$ projection operator from $L^{2}(K)$ onto $C_{K}^{E Q}$. Define

$$
C_{h}^{E Q}:=\left\{\psi \in L^{2}(\Omega)^{n},\left.\psi\right|_{K} \in C_{K}^{E Q} \text { for any } K \in \mathcal{T}_{h}\right\},
$$

and

$$
\left.P_{h}^{E Q}\right|_{K}=P_{K}^{E Q} \quad \text { for any } K \in \mathcal{T}_{h} \text {. }
$$

For the enriched rotated $Q_{1}$ element, it holds the following commuting property:

$$
\nabla_{h}\left(\Pi_{h} u\right)_{i}=P_{h}^{E Q} \nabla u_{i}, \quad i=1, \cdots, n,
$$

where $\left(\Pi_{h} u\right)_{i}$ and $u_{i}$ are the $i$-th components of $\Pi_{h} u$ and $u$, respectively. Such a commuting property which is proved in [14] for two dimension, in [9] for any dimension for the scale case, which can be adopt to the vector case.

For the enriched Crouzeix-Raviart element, we introduce the following space, for any $K \in \mathcal{T}_{h}$,

$$
C_{K}^{E C R}=\left(\begin{array}{c}
a_{11}+a_{0} x_{1} \\
a_{21}+a_{0} x_{2} \\
\cdots \\
a_{n 1}+a_{0} x_{n}
\end{array}\right),
$$

for $a_{11}, a_{21}, \cdots, a_{n 1}, a_{0} \in \mathbb{R}$. Let $P_{K}^{E C R}$ be the $L^{2}$ projection operator from $L^{2}(K)$ onto $C_{K}^{E C R}$. Define

$$
C_{h}^{E C R}:=\left\{\psi \in L^{2}(\Omega)^{n},\left.\psi\right|_{K} \in C_{K}^{E C R} \text { for any } K \in \mathcal{T}_{h}\right\},
$$

and

$$
\left.P_{h}^{E C R}\right|_{K}=P_{K}^{E C R} \quad \text { for any } \quad K \in \mathcal{T}_{h} .
$$

For the enriched Crouzeix-Raviart element, a similar argument of (5.8) is able to show the following commuting property:

$$
\nabla_{h}\left(\Pi_{h} u\right)_{i}=P_{h}^{E C R} \nabla u_{i}, \quad i=1, \cdots, n .
$$

Theorem 5.1. Let $(\lambda, u, p)$ and $\left(\lambda_{h}, u_{h}, p_{h}\right)$ be eigenpairs of (2.1) and (3.5), respectively. Then,

$$
\left\|u-u_{h}\right\|_{0} \lesssim(1+d) h^{\sigma}\left(\left(1+\lambda h^{2}\right)\left\|\nabla_{h}\left(u-u_{h}\right)\right\|_{0}+\inf _{q_{h} \in Q_{h}}\left\|p-q_{h}\right\|_{0}\right) .
$$


Proof. Let $f=\lambda u$ in (4.1) and (4.2), which implies that

$$
\left(u_{f}, p_{f}\right)=(u, p) \quad \text { and } \quad\left(u_{f, h}, p_{f, h}\right)=\left(\mathcal{P}_{h} u, \mathcal{P}_{h} p\right) .
$$

Further we let $\left.w\right|_{K}=\left.\Pi_{h} \lambda u\right|_{K}$ for any $K \in \mathcal{T}_{h}$ in Theorem 4.1. Then it follows from Theorem $4.1,(5.7),(5.8)$, and (5.9) that

$$
\left\|\nabla_{h}\left(u-\mathcal{P}_{h} u\right)\right\|_{0}+\left\|p-\mathcal{P}_{h} p\right\|_{0} \lesssim\left(1+\lambda h^{2}\right)\left\|\nabla_{h}\left(u-u_{h}\right)\right\|_{0}+\inf _{q_{h} \in Q_{h}}\left\|p-q_{h}\right\|_{0} .
$$

The desired result follows from Lemma 5.1, and (5.5).

We need the following error identity from $[8,20]$.

Lemma 5.2. Let $(\lambda, u, p)$ and $\left(\lambda_{h}, u_{h}, p_{h}\right)$ be eigenpairs of (2.1) and (3.5), respectively. It holds that

$$
\begin{aligned}
& \lambda-\lambda_{h} \\
= & \left\|\nabla_{h}\left(u-u_{h}\right)\right\|_{0}^{2}-\lambda_{h}\left\|u-u_{h}\right\|_{0}^{2}-2 \lambda_{h}\left(u-\Pi_{h} u, u_{h}\right) \\
& +2 a_{h}\left(u-\Pi_{h} u, u_{h}\right)-2 b_{h}\left(\Pi_{h} u, p_{h}\right) .
\end{aligned}
$$

Theorem 5.2. Let $(\lambda, u, p)$ and $\left(\lambda_{h}, u_{h}, p_{h}\right)$ be eigenpairs of (2.1) and (3.5), respectively. Suppose that

$$
1-\epsilon^{2}(h)>0 \quad \text { and } \quad\left\|\nabla_{h}\left(u-u_{h}\right)\right\|_{0} \geq \delta(h) /\left(1-\epsilon^{2}(h)\right) .
$$

Then

$$
\lambda \geq \lambda_{h}
$$

where

$$
\epsilon^{2}(h):=C_{1} \lambda_{h}(1+d)^{2}\left(1+\lambda h^{2}\right)^{2} h^{2 \sigma},
$$

and

$$
\delta^{2}(h)=C_{2} \sum_{K \in \mathcal{T}_{h}}\left(\lambda_{h}^{2} h_{K}^{4}\|\nabla u\|_{0, K}^{2}+\lambda_{h}(1+d)^{2} h^{2 \sigma} h_{K}^{2 \sigma}|p|_{\sigma, K}^{2}\right),
$$

provided that $p \in H^{\sigma}(\Omega)$.

Proof. We need to analyze the terms on the right-hand side of (5.12). It follows from (5.8) and (5.9) that both the fourth and fifth terms vanish. Let $\Pi_{0}$ be the piecewise constant $L^{2}$ projection operator with respect to $\mathcal{T}_{h}$. The definition of $\Pi_{h}$, the elementwise Poincare inequality, and (5.7) yield

$$
\begin{aligned}
& 2 \lambda_{h}\left(u-\Pi_{h} u, u_{h}\right)=2 \lambda_{h}\left(u-\Pi_{h} u, u_{h}-\Pi_{0} u_{h}\right) \\
\lesssim & \sum_{K \in \mathcal{T}_{h}} h_{K}^{2}\left\|\nabla\left(u-\Pi_{h} u\right)\right\|_{0, K}\left(\left\|\nabla\left(u_{h}-u\right)\right\|_{0, K}+\|\nabla u\|_{0, K}\right) .
\end{aligned}
$$

The final result follows from (5.10). 


\section{Numerical experiments}

In this section, we give the first six eigenvalues of Stokes eigenvalue problem on unit square by enriched rotated $Q_{1}$ element and enriched Crouzeix-Raviart element.

Table 1: The eigenvalues and the orders of convergence on a unit square domain, by enriched rotated $Q_{1}$ element.

\begin{tabular}{||c|cc|cc|cc||}
\hline level & $\lambda_{1}$ & $h^{n}$ & $\lambda_{2}$ & $h^{n}$ & $\lambda_{3}$ & $h^{n}$ \\
\hline 2 & $0.4167 \mathrm{E}+02$ & 0.00 & $0.4167 \mathrm{E}+02$ & 0.00 & $0.4167 \mathrm{E}+02$ & 0.00 \\
3 & $0.4714 \mathrm{E}+02$ & 1.04 & $0.7811 \mathrm{E}+02$ & 1.85 & $0.7811 \mathrm{E}+02$ & 1.85 \\
4 & $0.5063 \mathrm{E}+02$ & 1.60 & $0.8783 \mathrm{E}+02$ & 1.71 & $0.8783 \mathrm{E}+02$ & 1.71 \\
5 & $0.5188 \mathrm{E}+02$ & 1.88 & $0.9094 \mathrm{E}+02$ & 1.86 & $0.9094 \mathrm{E}+02$ & 1.86 \\
6 & $0.5223 \mathrm{E}+02$ & 1.97 & $0.9182 \mathrm{E}+02$ & 1.96 & $0.9182 \mathrm{E}+02$ & 1.96 \\
7 & $0.5231 \mathrm{E}+02$ & 2.00 & $0.9205 \mathrm{E}+02$ & 1.99 & $0.9205 \mathrm{E}+02$ & 1.99 \\
8 & $0.5234 \mathrm{E}+02$ & 1.96 & $0.9211 \mathrm{E}+02$ & 1.98 & $0.9211 \mathrm{E}+02$ & 1.98 \\
exact & $0.5234 \mathrm{E}+02$ & & $0.9212 \mathrm{E}+02$ & & $0.9212 \mathrm{E}+02$ & \\
\hline \hline level & $\lambda_{4}$ & $h^{n}$ & $\lambda_{5}$ & $h^{n}$ & $\lambda_{6}$ & $h^{n}$ \\
\hline 2 & $0.4167 \mathrm{E}+02$ & 0.00 & $0.4167 \mathrm{E}+02$ & 0.00 & $0.6857 \mathrm{E}+02$ & 0.00 \\
3 & $0.9537 \mathrm{E}+02$ & 1.40 & $0.1195 \mathrm{E}+03$ & 1.70 & $0.1310 \mathrm{E}+03$ & 1.45 \\
4 & $0.1166 \mathrm{E}+03$ & 1.51 & $0.1460 \mathrm{E}+03$ & 2.10 & $0.1623 \mathrm{E}+03$ & 2.94 \\
5 & $0.1249 \mathrm{E}+03$ & 1.84 & $0.1519 \mathrm{E}+03$ & 1.84 & $0.1655 \mathrm{E}+03$ & 1.61 \\
6 & $0.1273 \mathrm{E}+03$ & 2.10 & $0.1535 \mathrm{E}+03$ & 1.95 & $0.1666 \mathrm{E}+03$ & 1.89 \\
7 & $0.1280 \mathrm{E}+03$ & 2.84 & $0.1540 \mathrm{E}+03$ & 1.99 & $0.1669 \mathrm{E}+03$ & 1.97 \\
8 & $0.1282 \mathrm{E}+03$ & 0.84 & $0.1541 \mathrm{E}+03$ & 1.99 & $0.1670 \mathrm{E}+03$ & 1.99 \\
exact & $0.1281 \mathrm{E}+03$ & & $0.1541 \mathrm{E}+03$ & & $0.1670 \mathrm{E}+03$ & \\
\hline
\end{tabular}

It is clear from Table 1 that all eigenvalues converge from below. The computer error for $\lambda_{4}$ on the eighth-level grid is too big to violate the theory of lower bound.

Next, we use the enriched Crouzeix-Raviart element to compute the Stokes' eigenvalues, on uniform triangular grids where each square is cut by its upper-left to lower-right diagonal line. It is clear from Table 2 that all discrete eigenvalues converge from below.

\section{Acknowledgements}

The author would like to thank Prof. Shangyou Zhang for helping the numerical experiments. The author was supported by the NSFC under Grants Nos. 11571023 and 11401015.

\section{References}

[1] M. G. ARMentano AND R. G. DuRAn, Asymptotic lower bounds for eigenvalues by nonconforming finite element methods, ETNA, 17 (2004), pp. 93-101. 
Table 2: The eigenvalues and the orders of convergence on a unit square domain, by enriched Crouzeix-Raviart element.

\begin{tabular}{||c|cc|cc|cc||}
\hline level & $\lambda_{1}$ & $h^{n}$ & $\lambda_{2}$ & $h^{n}$ & $\lambda_{3}$ & $h^{n}$ \\
\hline 2 & $0.2648 \mathrm{E}+02$ & 0.00 & $0.2702 \mathrm{E}+02$ & 0.00 & $0.3199 \mathrm{E}+02$ & 0.00 \\
3 & $0.4308 \mathrm{E}+02$ & 1.48 & $0.5497 \mathrm{E}+02$ & 0.81 & $0.6045 \mathrm{E}+02$ & 0.92 \\
4 & $0.4956 \mathrm{E}+02$ & 1.73 & $0.8093 \mathrm{E}+02$ & 1.73 & $0.8203 \mathrm{E}+02$ & 1.65 \\
5 & $0.5159 \mathrm{E}+02$ & 1.88 & $0.8917 \mathrm{E}+02$ & 1.92 & $0.8940 \mathrm{E}+02$ & 1.89 \\
6 & $0.5215 \mathrm{E}+02$ & 1.96 & $0.9137 \mathrm{E}+02$ & 1.97 & $0.9143 \mathrm{E}+02$ & 1.97 \\
7 & $0.5230 \mathrm{E}+02$ & 1.99 & $0.9194 \mathrm{E}+02$ & 1.99 & $0.9195 \mathrm{E}+02$ & 1.99 \\
8 & $0.5233 \mathrm{E}+02$ & 1.98 & $0.9208 \mathrm{E}+02$ & 1.99 & $0.9208 \mathrm{E}+02$ & 1.99 \\
exact & $0.5234 \mathrm{E}+02$ & & $0.9212 \mathrm{E}+02$ & & $0.9212 \mathrm{E}+02$ & \\
\hline \hline level & $\lambda_{4}$ & $h^{n}$ & $\lambda_{5}$ & $h^{n}$ & $\lambda_{6}$ & $h^{n}$ \\
\hline 2 & $0.3200 \mathrm{E}+02$ & 0.00 & $0.3849 \mathrm{E}+02$ & 0.00 & $0.6857 \mathrm{E}+02$ & 0.00 \\
3 & $0.7461 \mathrm{E}+02$ & 0.85 & $0.7666 \mathrm{E}+02$ & 0.58 & $0.8252 \mathrm{E}+02$ & 0.22 \\
4 & $0.1110 \mathrm{E}+03$ & 1.64 & $0.1235 \mathrm{E}+03$ & 1.34 & $0.1410 \mathrm{E}+03$ & 1.70 \\
5 & $0.1234 \mathrm{E}+03$ & 1.86 & $0.1458 \mathrm{E}+03$ & 1.88 & $0.1599 \mathrm{E}+03$ & 1.88 \\
6 & $0.1270 \mathrm{E}+03$ & 2.05 & $0.1520 \mathrm{E}+03$ & 1.97 & $0.1652 \mathrm{E}+03$ & 1.96 \\
7 & $0.1279 \mathrm{E}+03$ & 2.49 & $0.1536 \mathrm{E}+03$ & 1.99 & $0.1666 \mathrm{E}+03$ & 1.99 \\
8 & $0.1281 \mathrm{E}+03$ & 2.57 & $0.1540 \mathrm{E}+03$ & 2.00 & $0.1669 \mathrm{E}+03$ & 2.00 \\
exact & $0.1281 \mathrm{E}+03$ & & $0.1541 \mathrm{E}+03$ & & $0.1670 \mathrm{E}+03$ & \\
\hline
\end{tabular}

[2] S. BRENNER, Poincaré-Friedrichs inequality for piecewise $H^{1}$ functions, SIAM J. Numer. Anal., 41 (2003), pp. 306-324.

[3] S. C. BRENNER AND L. R. SCOTT, The Mathematical Theorey of Finite Element Methods, Springer-Verlag, 1996.

[4] F. BReZzI AND M. Fortin, Mixed and Hybrid Finite Element Methods, Springer-Verlag, New York, 1991.

[5] C. CARSTENSEN AND J. HU, A unifying theory of a posteriori error control for nonconforming finite element methods, Numer. Math., 107 (2007), pp. 473-502.

[6] T. GUDI, A new error analysis for discontinous finite element methods for linear problems, Math. Comput., 79 (2010), pp. 2169-2189.

[7] Q. HOnG, H. XIE, M. YUE AND N. ZHANG, Fully computable error bounds for eigenvalue problem, Int. J. Numer. Anal. Model., 15(1-2) (2016), pp. 260-276

[8] J. HU AND Y. Q. HUANG, Lower bounds for eigenvalues of the Stokes operator, Adv. Appl. Math. Mech., 5 (2013), pp. 1-18.

[9] J. HU, Y. Q. HUANG AND Q. LIN, Lower bounds for eigenvalues of elliptic operators by nonconforming finite element methods, J. Sci. Comput., 61 (2014), pp. 196-221.

[10] J. HU, Y. Q. HUANG AND H. M. SHEN, The lower approximation of eigenvalue by lumped mass finite element methods, J. Comput. Math., 22 (2004), pp. 545-556.

[11] JUn Hu, Rui MA AND ZHONGCI SHI, A new a priori error estimate of nonconforming finite element methods, Sci. China Math., 57 (2014), pp. 887-902.

[12] XIA JI, YINGXIA XI AND HEHU XIE, Nonconforming finite element method for the transmission eigenvalue problem, Adv. Appl. Math. Mech., 9 (2017), pp. 92-103.

[13] SEUnGwOO LEE, DO YOUNG KWAK AND IMBO SIM, Immersed finite element method for eigenvalue problems in elasticity, Adv. Appl. Math. Mech., 10 (2018), pp. 424-444.

[14] Y. A. LI, Lower approximation of eigenvalue by the nonconforming finite element method, Math. 
Numer. Sin., 30 (2008), pp. 195-200.

[15] Y. A. LI, A posteriori error analysis of nonconforming methods for the eigenvalue problem, J. Syst. Sci. Complex., 22 (2009), pp. 495-502.

[16] Y. A. LI, New error estimates of nonconforming finite element methods for the Poisson Problem with low regularity solution, Adv. Appl. Math. Mech., 6 (2014), pp. 179-190.

[17] Q. LIN, H. T. HuANG AND Z. C. LI, New expansions of numerical eigenvalues for $-\Delta u=\lambda \rho u$ by nonconforming elements, Math. Comput., 77 (2008), pp. 2061-2084.

[18] Q. LIN AND J. LIN, Finite Element Methods: Accuracy and Improvements, Science Press, Beijing, 2006.

[19] Q. LIN, L. TOBISKA AND A. ZHOU, On the superconvergence of nonconforming low order finite elements applied to the Poisson equation, IMA J. Numer. Anal., 25 (2005), pp 160-181.

[20] Q. LIN, H. H. XIE, F. S. LUO AND Y. LI, Stokes eigenvalue approximations from below With nonconforming finite element methods, Math. Prac. Theory, 40(19) (2010), pp. 157-168.

[21] Q. LIN, H. XIE AND J. XU, Lower bounds of the discretization error for piecewise polynomials, Math. Comput., 83 (2014), pp. 1-13.

[22] H. P. LIU AND N. N. YAN, Four finite element solutions and comparison of problem for the poisson equation eigenvalue, Chinese J. Numer. Meth. Comput. Appl., 2 (2005), pp. 81-91.

[23] F. LUO, Q. LIN AND H. XIE, Computing the lower and upper bounds of Laplace eigenvalue problem: by combining conforming and nonconforming finite element methods, Sci. China Math., 55 (2012), pp. 1069-1082.

[24] S. P. MAO AND Z. C. SHI, On the error bounds of nonconforming finite elements, Sci. China Math., 53 (2010), pp. 2917-2926.

[25] R. RANNACHER, Nonconforming finite element methods for eigenvalue problems in linear plate theory, Numer. Math., 33 (1979), pp. 23-42.

[26] L. R. SCOTT AND S. Y. ZHANG, Finite element interpolation of nonsmooth functions satisfying boundary conditions, Math. Comput., 54 (1990), pp. 483-493.

[27] G. Strang And G. Fix, An Analysis of the Finite Element Method, Prentice-Hall, 1973.

[28] R. Verfürth, A Review of a Posteriori Error Estimation and Adaptive Mesh-Refinement Techniques, Wiley-Teubner, 1996.

[29] Y. D. YANG, Q. LIN, H. BI, AND Q. LI, Lower eigenvalues approximation by Morley elements, Adv. Comput. Math., 36 (2012), pp. 443-450.

[30] Y. D. YANG, Z. M. ZHANG AND F. B. LIN, Eigenvalue approximation from below using nonforming finite elements, Sci. China Math., 53 (2010), pp. 137-150.

[31] Z. ZHANG, Y. YANG AND Z. CHEN, Eigenvalue approximation from below by Wilson's elements, Chinese J. Num. Math. Appl., 29 (2007), pp. 81-84.

[32] O. C. Zienkiewicz AND Y. K. CheunG, The Finite Element Method in Structrural and Continuum Mechanics, New York: McGraw-Hill, 1967. 